crute s91:35-274

UCRL - 100890

Preprint

UCRL--100890

DE90 006990

\title{
Relativistic Klystron Wakefields
}

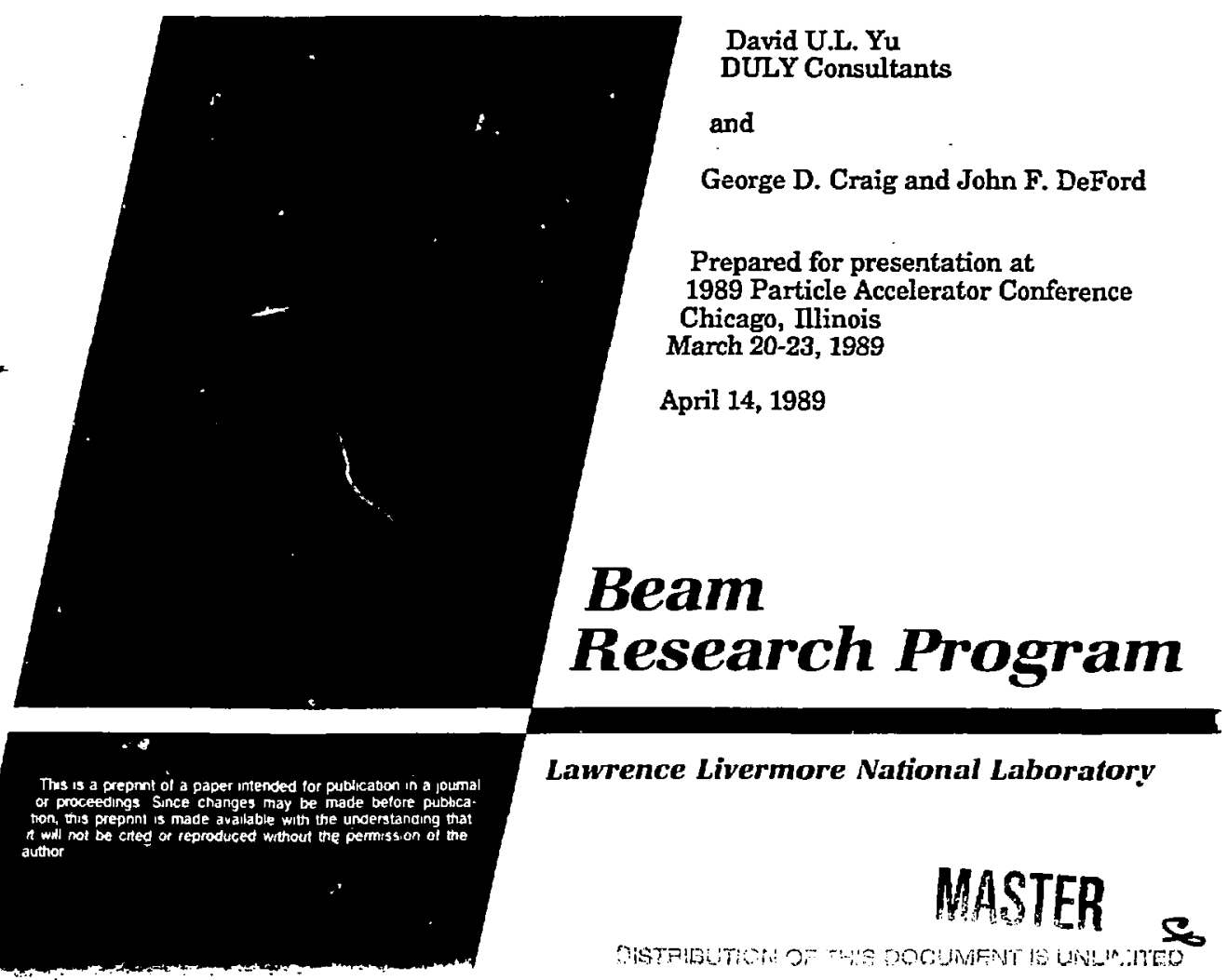




\section{DISCLAIMIER}

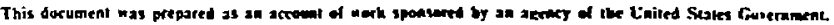

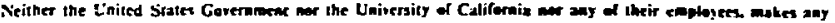

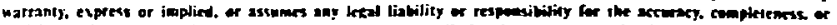

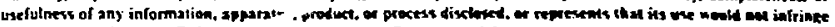

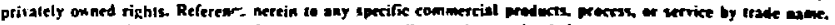

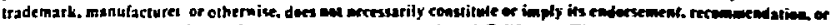

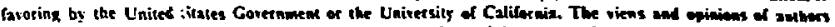

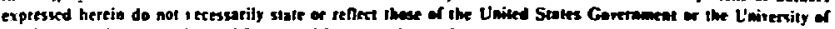

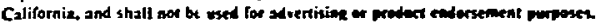




\title{
RELATIVISTIC KLYSTRON WAKEFIELDS*
}

\author{
David U.L. Yu \\ DULY Consultants \\ Rancho Palos Ficdes, CA 90732 \\ and \\ George D. Craig and John F. DeFord \\ Lawresce Livermore National Laboratory \\ Livermore, CA 9450
}

\section{Abstract}

Monopole, dipole and quadrupole wake potentials are calculated for two cavities in a standing-wave relativistic k]ystron, using two jndependent programs, TBCI and AMOS. Reflections from model terminations which may distort bng-range wakes can be mitigated either by using a very long pipe or by using absorptive materials at the pipe boundaries.

\section{Introduction}

In order for the relativistic klystron (RK) to fully achieve its potential as a high-gradient power souree at bigh frequencies for the next generation of linear colliders and compaci accelerators, it is important to control the wakefields produced by the electron bunches in the driver beam. The minimization of inergy spread and transverse beam breakup is particularly relevant for two beam accelerator schemes [1] in which reacceleration of the electron beam is an integral part. Accurate calctlations of the wakefields produced by electrons passing through various cavities are essential for investigation into bean stabiliiy problems. In this paper, we bave calculated the monppole, dipole and quadrupole wake potentials for typical relativistic klystron cavities. Calculations of the wakefields for the reacceleration cavities as well as bearn dynamics are currently in progress and will ie reported in subsequent papers.

We have chosen for our study here two standing-wave cavities used in the recent SIA experiment [2] performed at the Accelerator Research Center (ARC) at Livermore by the SLAC/LLNL/LEL collaboration. The beam pipe radius of the SL4 relativistic klystron is $7.0 \mathrm{~mm}$ for the first four cavities, and narrows downs to $4.6 \mathrm{~mm}$ for the penultimate and output cavities in order to extract power at $11.4 \mathrm{GHz}$ from a subharmonic drive. The lowest dipole modes of the first four SL4 cavities have frequencies above the cutoff frequency of the $T E_{11}$ mode in the beam pipe; while those for the last two cavities have frequencies below cutoff. Wakefields with dominant frequencies above cutoff are darnped as they exit the cavity region and propagate into the beam pipe. Long-range walefields with dominant frequencies below cutoff, on the other hand, ring for a long time as they are trapped in the region near the cavity.

In order to verify the numerical aceuracy of our modelg, we compare the wike potentials fens two independent computer programs, TBCI $\{3]$ and AMOS [.\}]. AMOS is a newly developed finite difference code which solvos Maxwell's equations in the time domain for cylindrically symmetric structures with linear material properties. The tesults of bot $h \mathrm{TBCI}$ and AMOS agree very well with cach other for a varicty of test cases. However, for wakes with frequencies above cutof, both prograns calculated spurious reflections from open boundaries of the beam pipe. These can be eliminated using long pipes, iut at the expense of long computer nun time. We have employed a nethod [5] in the AMOS code of using lossy dielectries with finite conductivities at the open ends of the bearn pipe to absorb the outgoing waves. We are able to show that a significant part of the spurious reflections at the open ends can be mitigated with tilis renttod.
Wakefields of the SL4 Relativistic Kilustron Cavities

The wake potential witnessed by a unit charge at a distance s behind a bunch of total charge $Q^{\prime}$ can be expressed in a multipole expansion as:

$$
\begin{aligned}
\vec{W}(s) & =\left.\frac{1}{Q^{r}} \int_{-\infty}^{\infty} d z(\vec{E}+\vec{v} \times \vec{B})\right|_{t=\frac{. t}{c}} \\
& =\frac{-\partial w_{0}(s)}{\partial s} z+w_{1}(s) r^{\prime} \dot{x}+\cdots
\end{aligned}
$$

where $\mathrm{z}$ is the bean axis, $\mathrm{r}^{\prime}$ is the radial displacement of the center of the bun:h from the aris, and $v$ is the velocity of - rigid bunch.[6] 'The first term in the expansion corresponds to the lonsitudinal monopole wake. The second term corresponds to the transverse dipole wake; and so on. Taking the Fourier transforms of the wakes, the longitudinal and transverse impedances are defined as:

$$
\begin{gathered}
Z_{10}(\omega)=\frac{1}{c} \int_{-\infty}^{\infty} d s \frac{\partial w_{0}}{\partial s} e^{i \omega \nu / c} \\
Z_{11}(\omega)=\frac{-i}{c} \int_{-\infty}^{\infty} d s w_{1}(s) e^{i \omega s / c}
\end{gathered}
$$

Figure 1 shows a TBCI model of cavity 3 in the SLA re:ativistic klystron. The $7 \mathrm{~mm}$ tean pipe radius gives a cutoff frequency of $16.4 \mathrm{GH}_{2}$ for the $\mathrm{TM}_{01}$ mode, and $12.6 \mathrm{GHz}$ for the $T E_{11}$ mode. Figures 2 to 4 are the monopole, dipole and quadrupole wakes for this cavity calculated with TBCI. These figures show the bunch shape, the longitudinal wake, the transverse wake and the aximuthal wake on a relative scale. The model for cavity 5 looks similar to Fig. 1. In this case, the 4.6

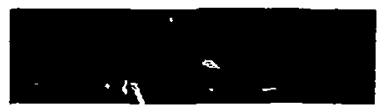

Fig. 1 TBCI model of SL4 relativistic klystron.

$\mathrm{mm}$ bean pipe radius gives a cutoff frequency of $25.0 \mathrm{GHz}$ for the $T M_{01}$ mode, and $19.1 \mathrm{GHz}$ for the $T E_{11}$ mode. Figures 5 to 7 are the wake potentials for this cavity calculated with TBCI. The beating seen in Fig. 7 is apparently caused by two quadrupole modes with frequencies very close to each otber, as a result of the dimensions chosen for the nose and the gap of this cavity.

From these figures, it is seen that the dominast frequencies of the RK wake potentials are below cutoff, except for the dipole wakes (Fig. 3) for cavity 3 which are above cutoff and as a result are strongly daraped as the felds propagate down the beam pipe. In this special case, as noted earlier [7], the 


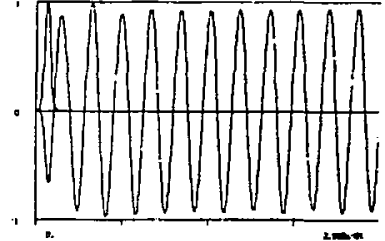

Fig. 2 SL4 cavity 3 monopulse wake for 0.3 neters.

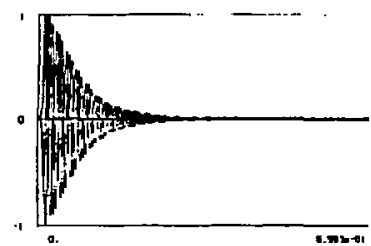

Fig. 3 SLA cavity ? dipole wakes for 0.7 meters using a $70 \mathrm{~cm}$ beam pipe.

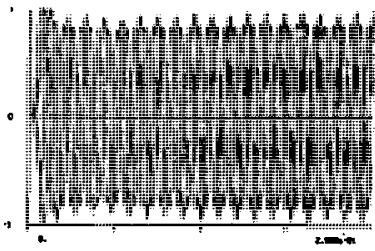

Fig. 4 SL 4 cavity 3 quadrupole wakes for 0.3 meters.

long range part of the wake is numerically dependent on the length of the model beam pipe. Spurious reflections from the open ends of the beam pipe can be eliminated in principle by making the bearn pipe long enough so that for a given simulation time, reflected waves from the open ends would not have sufficient time to reach back to interfere with the wakes generated directly by the bunch. In Fig. 3 we have shown the dipole wakes calculated with TBCI for cavity 3 with the beam pipe extending $35 \mathrm{~cm}$ on either side of the cavity. (The cavity itself has a gap of only $3.5 \mathrm{~mm}$.) Reflections are effectively suppressed for this long pipe caiculation.

In practice, the necessity of modeling cavities with very long pipes in order to obtain accuracy for the long range wakes makes the problems cumbersomely large, and computation times unrealistically long. We are therffore motivated to find alternative wazs to obtain accurate wakefields for cavity design and beam dynamics calculations. We examine next a new wakefield code AMOS [4] which allows one to design an absorptive load and shorten the beam pipe.

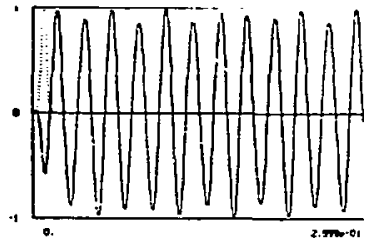

Fig. 5 SLA cavity 5 monopole wake for 0.3 meters.

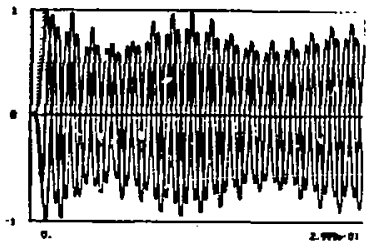

Fig. 6 SLt cavity 5 dipole wakes for 0.3 meters.

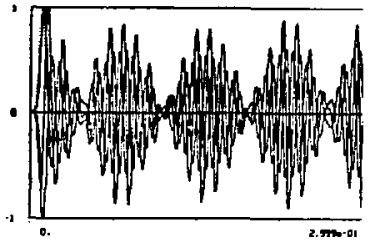

Fig. 7 SLA cavity 5 quadrupole wakes for 0.3 meters.

\section{Comparison of AMOS and TBCI}

The RK cavity dimensions are modified somewhat for the purpose of this comparison in order to accommodate the current limitations of the AMOS program. In particular, we have idealized the cavity model to eliminate curved surfaces and to move boundary lines of the structure to coincide with the ed ges of the uruform rcctangular meshes. Figure 8 shows schematically the regions (cross hatched) of the klystron and pussible resistive loads at the ends of the bearn pjpe. Using this model, we have calculated the moropole, dipole and quadrupole wake polentials for open or closed boundary conditions, and for varying lengths of the beara pipe. Results from AMOS and TBCl for the idealized models without the loads are in excellent agreement. Figure 9 shows, for instance, the dipole transverse wakes for a cavity with $7 \mathrm{~mm}$ radius calculated with the two programs. The two wakes averlay perfectly on this acale. Compared srith the long pipe calculation of Fig. 3 , these short 
pipe calculations also illustrate that when Maxwell's equations are solved with the first order radiative boundary conditions used in TBCI or AMOS, the wake potentials are sensitive to refections from the open bourdaries, as is evidenced by the broad secondary peak in Fig. 9.

Absomtion Model for Boundary Termination

We now use the AMOS code to investigate here a theoretical method [5] to mitigate reflections from terminations. The method uses the fact that lossy dielectrics can be used to absorb ri power. Instead of open pipes, we model the terminations with absorptive materials having $\epsilon_{r}=1$ but with a gradual increase of conductivity from zero (vacuum) to a maximum value, before capping off with perfect metal. A parabolic distribution of conductivities is used to simulate the effects of absorption over a distance of about one wavelength $(\lambda=2.1 \mathrm{~cm})$. Although only three regions are shown at each end of the pipe in Fig. 8, 120 regions with 60 absorptive materials are used in the actual calculations. The model for the $7 \mathrm{~mm}$ radius cavity consists of a total of 444 zones along the beam axis, 40 zones for the pipe radius, 20 zones across the gap, and 44 zones along the roof of the cavity. Each side of the square zone has a length of $0.175 \mathrm{~mm}$. In this model, the beam pipe extends only 3.69 con on each side of the cavity.

In Fig. 10, we plot the value of the secondary peak, which is a measure of the pistial retections, of tbe wake potential as s function of the maximun value of conductivity used in the absorption model. If the dielectrics have very small valueg of conductivities, they behave essentially like vacuum. Thus large reflections are expected from the metal boundaries. On the other hand, if the dielectrics have high conductivities, tbey behave like metals; and large reflections are also expected. For a fixed absorption length, there is an optimum value of the maximun conductivity which provides maximum absorption, and hence minimum secondary peak of the wake potential. For the $7 \mathrm{~mm}$ radius cavity, the "best" conductivity model has a waximum conductivity of $0.55 \mathrm{mho} / \mathrm{m}$ at the ends of the beam pipe. Ous sensitivity study of the parabolic absorption model has so far only varied the value of the maximum conductivity for a fixed absorption length. Effects of other functional forms of the absorption models and of the lengths of the absorption regions are open to further investigations.

-Work supported by DOE contract numbers DE-ACO387ER80529 (DULY) and W-7405-ENG-48 (LLNL).

[1] A.M. Sessler and S.S. Yu, Phys. Rev, Lett., 58, 2439 (1987).

[2] M.A. Allen et al., SLAC-PUB-4650, June 1988.

[3] T. Weiland, Nucl. Instr. Meth. 212, 13 (1983).

[4] J. DeFord, G. Craig and R. McLeod, Proc. 1989 PAC, Paper P10.

[5] M. Caplan, Ph.D. Thesis, UCLA (1086).

[6] P. B. Wilson, SLAC-PUB-4547, January 1989.

[7] T. Higo et al., SLAC/AP-70, AAS-37, Jene 1988.

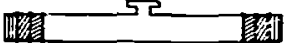

Fig. 8 Ideailized AMOS model of SLA cavity 3 with resistive loads at the beam pipe ends.

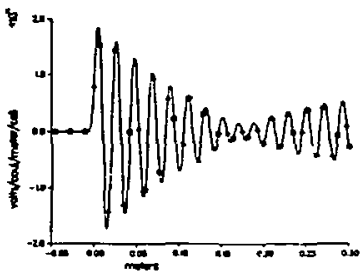

Fig. 9 Transverse dipole wakes from TBCI and AMOS for 0.3 meters for pipe length $=7.46 \mathrm{~cm}$.

Absorption Effects vs Conductivity

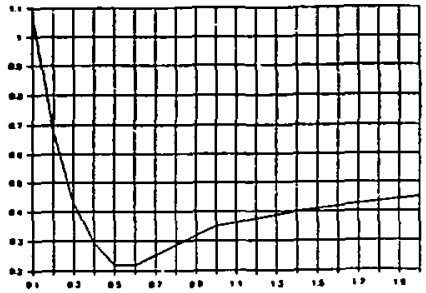

Fig. 10 Max. value of secondary peak vs max conductivity of dielectric load. 